\title{
Correlation between off-axis illumination and apodized phase-contrast: two complementary microscopic phase-imaging modes
}

\author{
Radek Pelc* \\ The Stentor Institute \\ Hostivice-Palouky 614 \\ CZ-25301 Praha-Západ, Czech Republic \\ and \\ Academy of Sciences \\ Institutes of Microbiology and Physiology \\ Biomedical Research Institutes Centre \\ K Výzkumným ústavům 1083 \\ CZ-14220 Praha 4-Krč, Czech Republic
}

\author{
Zdeněk Hostounský \\ The Stentor Institute \\ Hostivice-Palouky 614 \\ CZ-25301 Praha-Západ, Czech Republic
}

\section{Tatsuro Otaki}

Nikon Corporation

Optical Design Department

Core Technology Center

1-6-3 Nishi-Ohi, Shinagawa-ku

Tokyo 140-8601, Japan

\begin{abstract}
Microscopic images of biological phase specimens of various optical thickness, acquired under off-axis illumination and apodized/conventional phase-contrast are compared. The luminance profiles in appropriately filtered apodized phase-contrast images compare well with those in the original off-axis illumination images. The two unfiltered image types also yield similar results in terms of quasithree-dimensional surface (pseudo-relief) rendering, and thus are comparable in terms of the information contents (optical thickness map). However, the overall visual impression is very different as the visual cues to depth structure are present in the off-axis illumination images only. The comparison demonstrated in the present paper was made possible owing to apodization, which substantially reduces the "halo"/shade-off artifacts in the phase-contrast images. The results imply the possibility of combining the off-axis illumination and apodized phase-contrast imaging to examine specimens of medium optical thickness, in which the phase visualization capability of the two imaging modes substantially overlaps (e.g., larger cells or cell clusters). 12008 Society of Photo-Optical Instrumentation Engineers. [DOl: $10.1117 / 1.2966716]$
\end{abstract}

Keywords: apodization; depth perception; microscopy; phase-contrast; pseudo-relief; shape-from-shading; schlieren imaging; visual cues.

Paper 07145R received Apr. 17, 2007; revised manuscript received Jan. 11, 2008; accepted for publication Feb. 19, 2008; published online Oct. 29, 2008.

\section{Introduction}

Microscopic phase objects-those featuring no amplitude contrast that would otherwise make them visible even under a standard microscope illumination (i.e., axial bright field) can be visualized in various ways. The most common one is the phase-contrast imaging ${ }^{1,2}$ (1953 Nobel Prize to Frits Zernike $^{3}$ ). Other imaging modes capable of revealing the phase information include the differential interference contrast (e.g., after Nomarski ${ }^{4}$ ) and various schlieren illumination modes, ${ }^{5}$ the most common one being the Hoffman modulation contrast, ${ }^{6}$ its simpler form, ${ }^{7}$ and the off-axis (oblique, inclined, single-sideband, asymmetric) illumination. ${ }^{8}$ Various other microscopic schlieren imaging modes, essentially combinations of the abovementioned ones have been briefly listed elsewhere.

In phase objects, the luminance profiles in the off-axis illumination (or generally schlieren) image roughly correspond to those of the optical path difference gradients in the apparent direction of illumination; that is, perpendicular to the

\footnotetext{
*Address all correspondence to Radek Pelc, Institute of Physiology, Academy of Sciences, K Výzkumným ústavům 1083, CZ-14220 Praha 4-Krč, Czech Republic; Fax: +420-24106-2488; E-mail: radek.pelc@seh.oxon.org

A preliminary account of the results shown in this paper was previously presented: R. Pelc, Z. Hostounský, and T. Otaki, "On correlation between two phase-imaging modes: off-axis illumination and apodized phase-contrast," in Proc. Annu. Conf. Czechoslovak Microsc. Soc. (Mikroskopie 2008), p. 19 (2008).
}

edge of the schlieren (cut-off, relief) diaphragm; $;^{2,5,10}$ this dependency is not exactly linear. ${ }^{11}$

The optical path difference (OPD) equals $\left(n_{1}-n_{2}\right) t$, where $n_{1}$ and $n_{2}$ are the refractive indices of the medium and phase object, respectively, and $t$ is the object's (physical) thickness; $n_{2} t$ is optical thickness. The phase shift induced by the phase object equals $2 \pi \times \mathrm{OPD} / \lambda$. For example, if the medium is air $\left(n_{1}=1\right)$, OPD and phase shift are negative; the object is phase-retarding.

In ideal, artifact-free phase-contrast images, the dependency between the luminance and the OPD itself is nearly linear within certain OPD intervals. If the phase object is also sufficiently thin (OPD is only a fraction of wavelength), the luminance in each phase-contrast image pixel is also almost directly proportional to the OPD in a corresponding point in the specimen; this holds for both phase-retarding objects (OPD is positive, e.g., cells in water) in negative phasecontrast and phase-advancing objects (OPD is negative, e.g., gas vacuoles in cells) in positive phase-contrast. ${ }^{12-14}$ These situations can be easily documented with vector diagrams, ${ }^{3}$ whose usage was extensively described in Barer's papers, as shown elsewhere. ${ }^{12,13}$

In terms of the information contents, the off-axis illumination and artifact-free phase-contrast images can thus be regarded as more-or-less equivalent. Indeed, the fact that the

$1083-3668 / 2008 / 13(5) / 054067 / 12 / \$ 25.00$ (C) 2008 SPIE 
quasi-three-dimensional (pseudo-relief) effect inherent to the off-axis illumination (or generally schlieren) images can also be obtained by differentiating (directional gradient filtering) the phase-contrast ones has been known for a long time. Qualitative examples have been reported in both optical ${ }^{15-17}$ and electron ${ }^{18}$ microscopy.

However, the relationship between the off-axis illumination and phase-contrast images is generally difficult to document quantitatively. Such efforts have been inevitably hampered by the "halo"/shade-off artifacts inherently present in the phase-contrast images of thicker objects usually featuring large OPDs. In very thin objects, for example, tissue culture cells spread on a glass microslide or a Petri dish, this artifact is often small or even absent altogether, ${ }^{3,16}$ but the off-axis illumination (or, for example, Hoffman modulation contrast) imaging is rather insensitive in detecting the OPD ("phase") gradients in such cases. Indeed, the low sensitivity of schlieren imaging (where only the intensity of direct light is altered) was the very motivation behind the invention of the phase-contrast microscope, ${ }^{1,2}$ although the idea that is pivotal in this method-to selectively alter also the phase of the direct light—was reported much earlier. ${ }^{19}$

To bridge this gap between the two phase-imaging modes, we use off-axis illumination and a refined form of phasecontrast imaging, the apodized phase-contrast microscopy. The latter delivers reduced halo/shade-off artifact images by purely optical means ${ }^{14,20-28}$ (Fig. 1), that is, without the need for any postdetection processing ${ }^{17,29,30}$ that would otherwise have to be employed..$^{30}$ Apodized phase-contrast thus makes it possible to reliably visualize thicker phase specimens ${ }^{22,28}$ than a conventional phase-contrast.

The aim of the present paper is to document, in biological phase specimens of various optical thicknesses, the differences between the two imaging modes in terms of image information contents. Digital image filtering of apodized phase-contrast (artifact-free) images is employed and the effects of visual cues to depth structure in comprehension of quasi-3D (off-axis illumination) images are discussed.

Note: Because the media surrounding the phase objects under investigation were not altered during the observations, and the data presented here are of semiquantitative nature only, a more familiar term, "optical thickness" is often used instead of a more rigorous one, "optical path difference" (OPD). As stated previously, both of them are proportional to the object's physical thickness.

\section{Experimental Proceedings}

\subsection{Specimens}

Almost purely phase specimens were employed to evaluate the phase-visualization capability of the optical set-ups used:

1. Replicas in a transparent medium of plant leaf surfaces were prepared according to an adhesive method after Wolf, as described elsewhere. ${ }^{9}$ Briefly, a thin layer of an acrylate resin/ adhesive [xylene solution of butyl- and methylmethacrylate copolymer (Solakryl BMX, Draslovka a.s., Kolín, Czech Republic)] was spread on the leaf, left to harden, and peeled from the leaf surface by an adhesive transparent plastic foil (an ordinary sticky tape). A colorless nail varnish may also be used instead of the resin. The resulting replica was placed on a microslide together with the plastic foil. In this way, a permanent specimen was prepared. The plant species used were coachgrass, also known as quackgrass or dog grass [Agropyron repens (L.) Gould (Cyperales, Poaceae)]; and catchweed, also known as catchweed bedstraw, cleavers, or stickywilly [Galium aparine L. (Rubiales, Rubiaceae)].

2. Radula of the Spanish slug [Arion lusitanicus Mabille 1868 (Stylomatophora, Arionidae)] is a translucent chitinous ribbon in a slug's mouth. Its function is to rasp food with its indented surface (denticles or "teeth"). Radula preparation has been described elsewhere, ${ }^{31}$ and here we use another portion of the same specimen. Briefly, soft tissues of a dissected front portion of the slug's body were digested with a concentrated $\mathrm{NaOH}$ solution $(\sim 10 \%$ weight/volume $)$, and the radula thus isolated was mounted in air between a microslide and a coverslip.

3. Longitudinal section from the wood of Norway spruce [Picea abies (L.) Karst. (Pinales, Pinaceae)], embedded in Canada balsam has been obtained locally; a synonym for this species is Picea excelsa (Lamb.) Link.

\subsection{Microscopes}

All off-axis illumination images shown in Figs. 3-6 were acquired with an upright laboratory microscope, DN45-BH51 model (Lambda Praha, Prague, Czechoslovakia) equipped with a dedicated off-axis illumination Abbé-type condenser [numerical aperture (NA) 1.2], $\mathrm{RCH}-0128$ model with a built-in shifting relief diaphragm, as described in detail elsewhere. ${ }^{9,31,32}$ An achromatic objective, $\times 10 / 0.25$ was used.

The phase-contrast image of the slug's radula [Fig. 6(d)]) was acquired with another Lambda DN45-BH51 upright microscope fitted with a phase-contrast condenser (NA 1.25) normally supplied with DN45-BH47Ph microscope model. A planachromatic objective, Plan 10Ph/0.25 was used (conventional phase-contrast, negative type).

All above-mentioned photomicrographs were acquired with Praktica VLC3 camera (Pentacon, Dresden, Germany), on Fuji Superia 100 color print film, using a photographic eyepiece $(\mathrm{FU} \times 4)$. An ordinary (non-interference) green filter $(\lambda \sim 550 \mathrm{~nm})$ was used throughout.

Except for Fig. 6(d), all other phase-contrast images shown in Figs. 3-6 were acquired with the following Nikon (Tokyo, Japan) CFI achromatic objectives of DL ("dark low") series: $\times 10 / 0.25$ DL (conventional phase-contrast, positive type) and $\times 10 / 0.25$ ADL (apodized phase-contrast, positive type). A green interference filter $(\lambda=530 \mathrm{~nm})$ and a personal computer-controlled DS-Fil charge-coupled device camera were used throughout. All positive-type phase-contrast images shown in Figs. 3 and 4 and Figs. 5 and 6 were acquired on Nikon Eclipse inverted microscopes (TE2000-S and TS100-F models, respectively), each fitted with a halogen lamp $(100 \mathrm{~W}$ and $30 \mathrm{~W}$, respectively) and a (extra) long-workingdistance condenser (LWD, NA 0.52, working distance $30 \mathrm{~mm}$; and ELWD, NA 0.30, working distance $75 \mathrm{~mm}$, respectively) with a $\mathrm{Ph}-1$ annular aperture.

\subsection{Apodized Phase-Contrast}

The apodized phase-contrast microscope ${ }^{14,20-28}$ differs from a conventional one by two light-attenuating annuli 


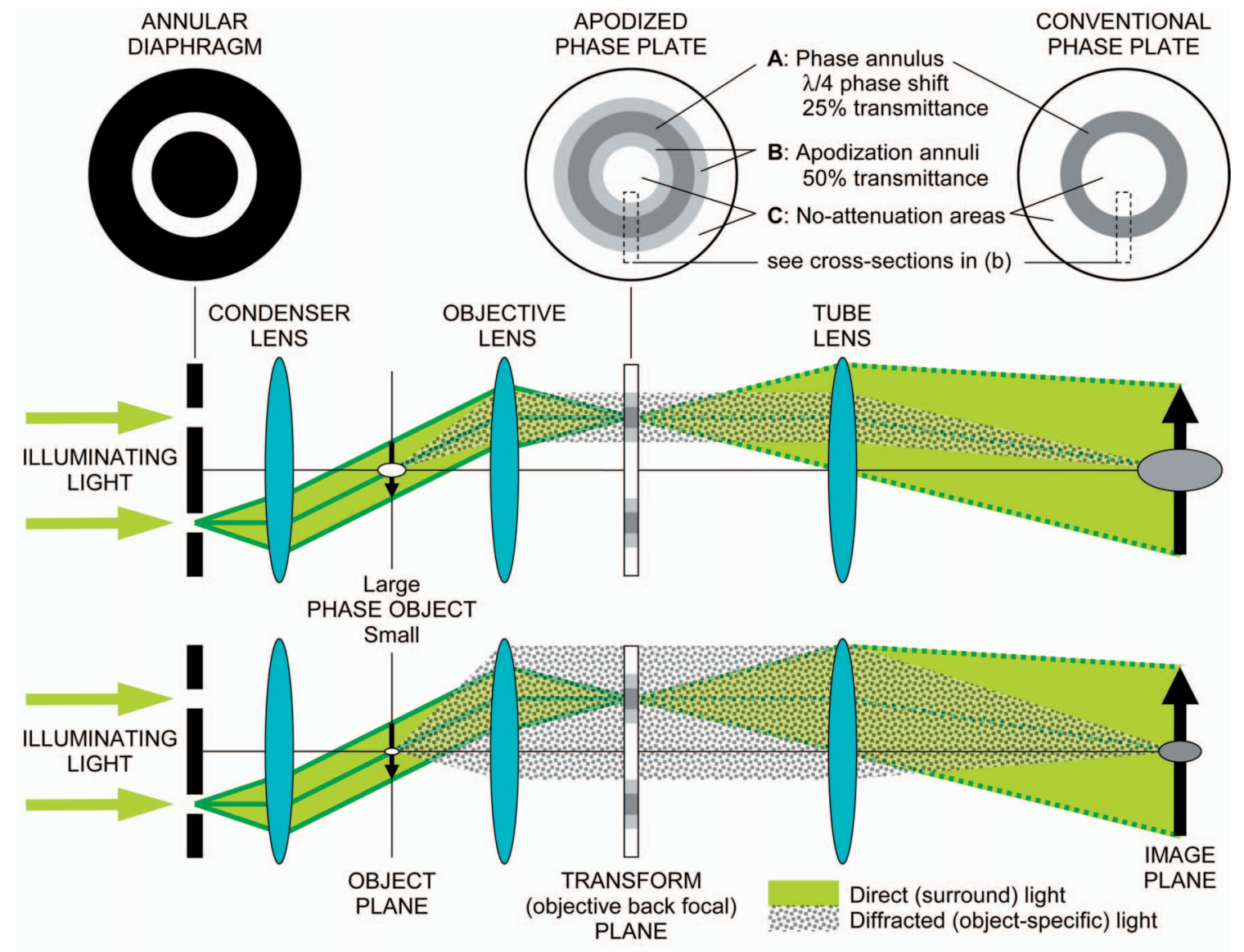

(a)

\section{Periodic objects}

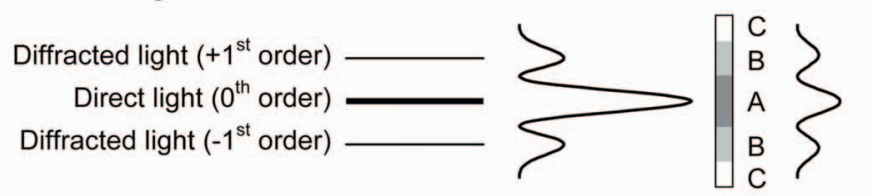

APODIZED PHASE-CONTRAST

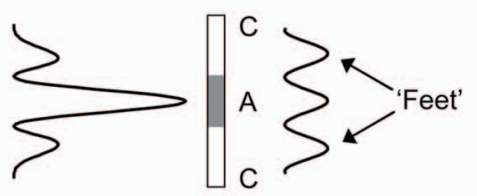

CONVENTIONAL PHASE-CONTRAST

(b)

Fig. 1 The principle of apodized phase-contrast microscopy (qualitative aspects only). Details on the theory can be found in the text, accompanying vector diagrams (Fig. 2) and an interactive online material (Ref. 28). (a) Optical setup. Instead of a conventional phase plate, an apodized phase plate is located at the transform (objective back focal) plane conjugate with the condenser's front focal plane hosting the annular diaphragm. Objects that are large in terms of lateral dimensions (thus diffracting light to small angles) are typically also thick in terms of OPD and so diffract rather strongly, causing profound halo/shade-off artifacts. Most of this light is attenuated by the apodization annuli (B) adjacent to the phase annulus (A). Small objects diffract light to wider angles; it passes mostly through the no-attenuation areas (C) and is largely unaffected by the phase annulus (A) and the apodization annuli (B). The image of the large/small colorless phase object is "optically stained" (light/dark gray); apodization somewhat reduces contrast in images of large thin objects but markedly supresses the halo/shade-off artifacts in images of large thick objects. (b) The principle of apodization (from Greek, meaning "foot removal") illustrated with a periodic object yielding a distinct diffraction pattern registering with the apodization annuli.

immediately adjacent to the phase annulus (Fig. 1). Its principle is based on apodization and can be briefly described as follows:

The angle of light diffracted by an object (e.g., a cell) decreases as the object's size (or, to be precise, its lateral dimensions) increases [Fig. 1(a)]. In large objects, a considerable fraction of diffracted light thus passes through the phase annulus that, however, should ideally be sanctioned to the direct light only. This brings about distortions in the luminance profiles in the images, called halo and shade-off artifacts; they surround and fill the object's image, respectively. The reason is that the diffracted light cannot fully contribute to image formation as it has been attenuated by the phase annulus. ${ }^{3,12,13}$ An analytical explanation of the halo/shade-off artifacts is possible either with Bessel functions $^{1,21}$ or, to some extent, with vector diagrams, ${ }^{3,12,13}$ 


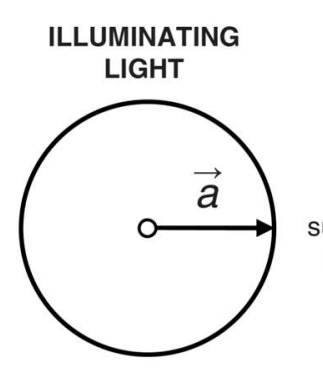

$\begin{array}{ll}\text { OBJECT } & \text { TRANSFORM } \\ \text { PLANE } & \text { PLANE }\end{array}$ surrounding the phase object

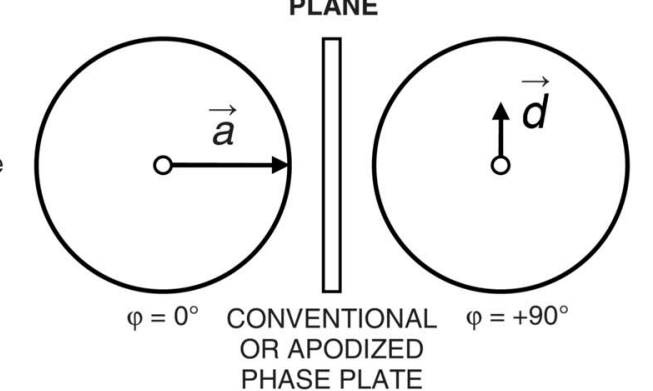

PHASE PLATE
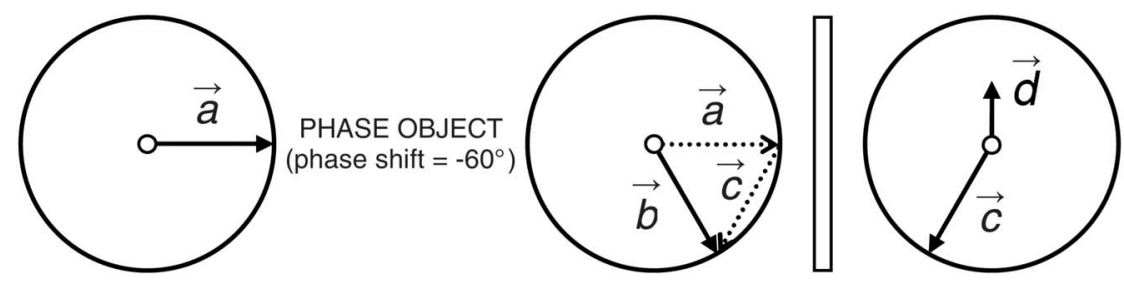

CONVENTIONAL

PHASE PLATE
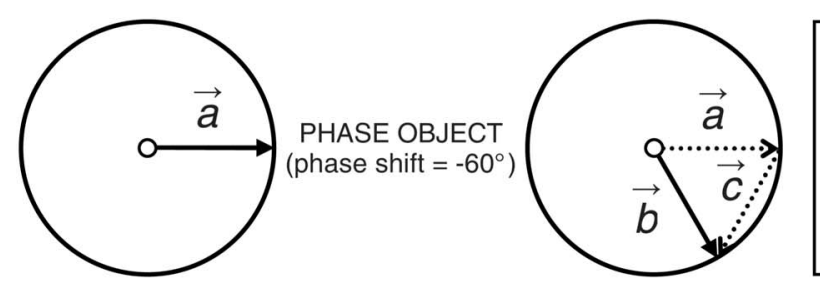

APODIZED

PHASE PLATE

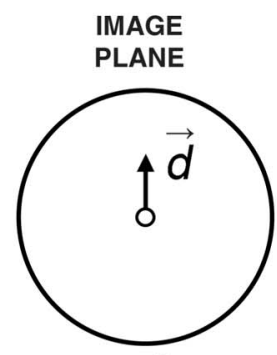

Amplitude $(\vec{d})=0.500$ Intensity $=|\vec{d}|^{2}=0.250$ (background light)

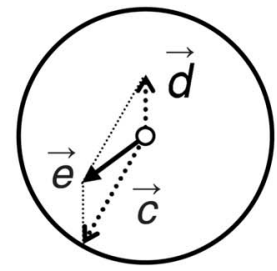

Amplitude $(\vec{e})=0.620$ Intensity $=|\vec{e}|^{2}=0.384$ ( $154 \%$ of background)

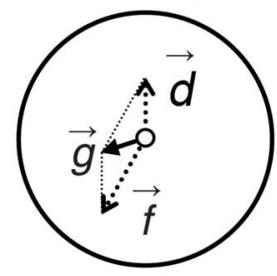

Amplitude $(\vec{g})=0.371$ Intensity $=|\vec{g}|^{2}=0.138$ ( $55 \%$ of background)

$\vec{a}=$ illuminating light; direct light through the specimen and the surrounding medium (unperturbed waves), $|\vec{a}|=1.00$

$\vec{b}=\vec{a}+\vec{c}=$ all light passing through the specimen, $\vec{b}=\vec{a} \mathrm{e}^{-i \pi / 3}\left(-60^{\circ}\right.$ phase shift)

$\vec{c}=$ diffracted light (perturbed wave)

$\vec{d}=\sqrt{0.25} \vec{a} e^{i \pi / 2}=$ background (surround) light in the image (direct light, after passing through the phase annulus that advances its phase by $90^{\circ}$ and reduces its intensity to $25 \%$ )

$\vec{e}=\vec{d}+\vec{c}=$ interfered light representing the object in the image (conventional phase-contrast, positive type)

$\vec{f}=\sqrt{0.50} \vec{c}=$ diffracted light, after passing through the apodization annuli that reduce its intensity to $50 \%$

$\vec{g}=\vec{d}+\vec{f}=$ interfered light representing the object in the image (apodized phase-contrast, positive type)

Fig. 2 Vector diagrams applied here to "calculate" luminance in the images of a nonabsorbing (transparent) phase-retarding object (-60-deg phase shift relative to the surrounding medium, or OPD $=-\lambda / 6$ ), in conventional and apodized phase-contrast (positive type in both cases). For simplicity it is assumed that all diffracted light passes through the apodization annuli and that the medium causes a negligible phase shift (e.g., air). Arrows represent light vectors ( $\vec{a}$ to $\vec{f})$, their length and angle indicating light wave amplitude $(A)$ and phase $(\varphi)$, respectively. Light intensity (luminance), $I=\left|A e^{i \varphi}\right|^{2}=A^{2}$. The strongest contrast is achieved when vectors $\vec{e}$ and $\vec{g}$ are as small as possible, that is, $|\vec{d}| \sim|\vec{c}|$ and $|\vec{d}| \sim|\vec{f}|$ (conventional and apodized phase-contrast, respectively). Note that in the conventional phase-contrast, the luminance in object's image is already greater $\left(|\vec{e}|^{2}=154 \%\right)$ than the background luminance $\left(|\vec{d}|^{2}=0.25=100 \%\right)$, in other words, it is giving rise to contrast reversal. In apodized phase-contrast, the object still appears darker $\left(|\vec{g}|^{2}=55 \%\right)$ than the background. Apodization in phase-contrast microscopy thus broadens the range of OPDs (broadly speaking, optical thicknesses) yielding unreversed contrast. As it attenuates the diffracted light, it is also instrumental in reducing the halo/shade-off artifacts.

which are also used in the present study (Fig. 2).

On the other hand, the amplitude of the diffracted light increases with the optical path difference (OPD) between the object and the surrounding medium (Fig. 2). The artifacts are thus insignificant in thin objects, regardless of their lateral dimensions (e.g., tissue culture cells spreading on a flat surface $^{3}$ ); the intensity of diffracted light is low. They only become prominent in those large objects that are also thick, in terms of OPD (e.g., cell clusters ${ }^{28}$ ). Such objects diffract light strongly, thus causing noticeable artifacts.

The 50\% transmittance apodization annuli decrease the relative contribution of the artifact-generating diffracted rays (i.e., those that have been absorbed by the phase annulus). In a simplified case of a periodic object with a particular spatial frequency, the \pm 1 diffraction maxima (side lobes or "feet") 
OFF-AXIS ILLUMINATION

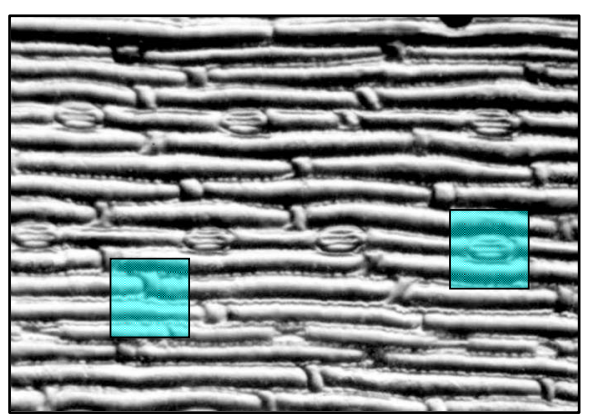

APODIZED PHASE-CONTRAST

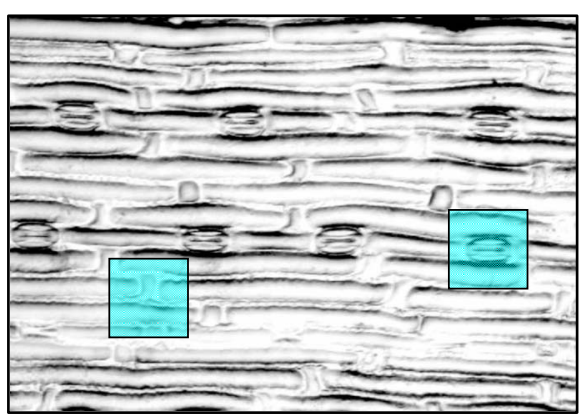

\section{CONVENTIONAL PHASE-CONTRAST}

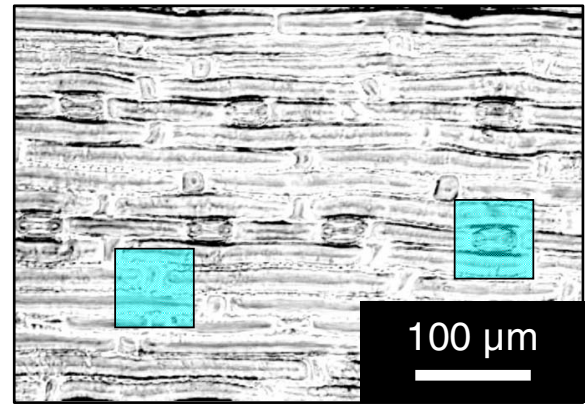

Fig. 3 Phase specimen with a uniform refractive index (leaf replica from coachgrass, Agropyron repens). Its optical and physical thickness profiles directly correspond. Three different microscopic phase-imaging modes are used. Note the strong relieflike impression in the off-axis illumination image, facilitating a direct visual 3D "reconstruction." Corresponding quasi-3-D surface rendering diagrams are shown in Video 1. The halo/shadeoff artifacts in the apodized phase-contrast image are substantially reduced compared with the conventional phase-contrast one. Phase-contrast type: positive. The two boxed motifs are shown in Fig. 4 at an expanded scale.

pass through the apodization annuli and are attenuated (feet removed, or apodized) to $50 \%$ [Fig. 1(b)].

The price that is paid for the artifact reduction in the apodized phase-contrast images is a lower maximum theoretically achievable contrast, which can be easily calculated ${ }^{25}$ or demonstrated with vector diagrams (Fig. 2). This effect is only significant in large objects; in small ones, light is diffracted to greater angles and most of it bypasses the apodization annuli [Fig. 1(a)]. In real images, however, the contrast may actually increase owing to apodization because the structural details are no longer masked by the halo/shade-off artifacts (Figs. 3-5 and Video 1).

\subsection{Images}

In Fig. 3, images of the same specimen area, acquired under different imaging modes, were aligned to match, at \pm 1 to 2 pixel precision $(720 \times 500$ images $)$, which translates to $\pm 1 \%$ to $2 \%$ of image width in selected cutout details shown in Fig. 4. This value is within the Rayleigh resolution limit of $0.61 \lambda / \mathrm{NA} \sim 1.3 \mu \mathrm{m}$ for green light used here. A somewhat smaller precision in aligning the images was afforded in Figs. 5 and 6 where no quantitative image analysis was carried out.
OFF-AXIS ILLUMINATION

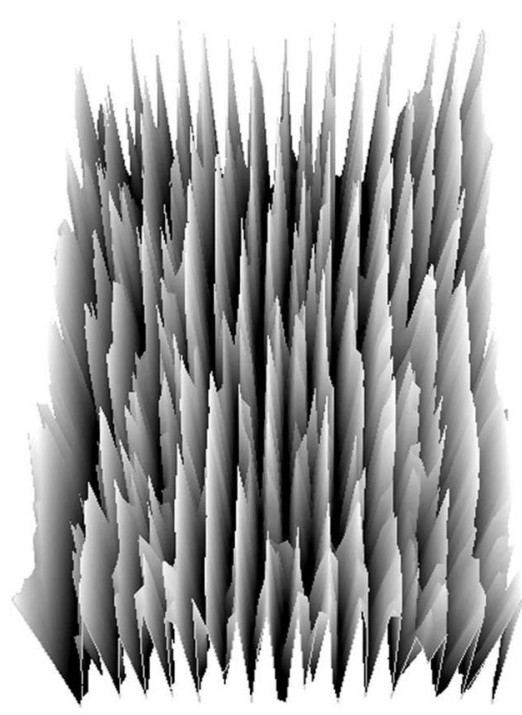

(a)
APODIZED PHASE-CONTRAST

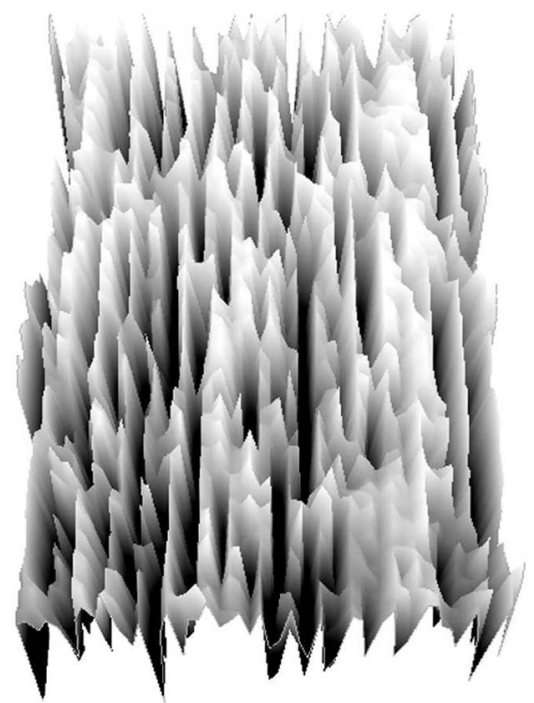

(b)

\section{CONVENTIONAL PHASE-CONTRAST}

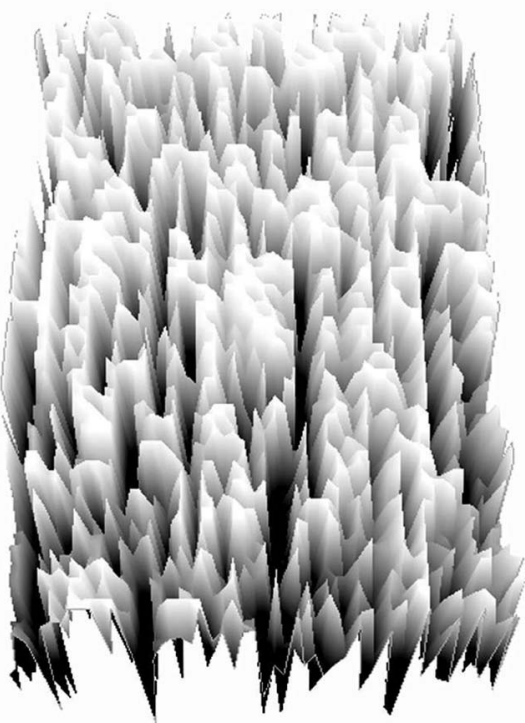

(c)

Video 1 Quasi-3D surface rendering of the images shown in Fig. 3 (tilted by 30 deg around the $x$ axis and 90 deg anticlockwise around the $z$ axis in 1 -deg increments). The information that can be extracted from the three image types is comparable, although the quasi-3D surface rendering starts failing in the conventional phase-contrast image, in which the structure is blurred by profound halo/shade-off artifacts; this is more apparent in the videos themselves. Video 1(a) (MOV, 2.5MB) [URL: http://dx.doi.org/10.1117/1.2966716.1]; Video 1(b) (MOV, 1.7MB) [URL: http://dx.doi.org/10.1117/1.2966716.2]; Video 1(c) (MOV, 1.8MB) [URL: http://dx.doi.org/10.1117/1.2966716.3]. 

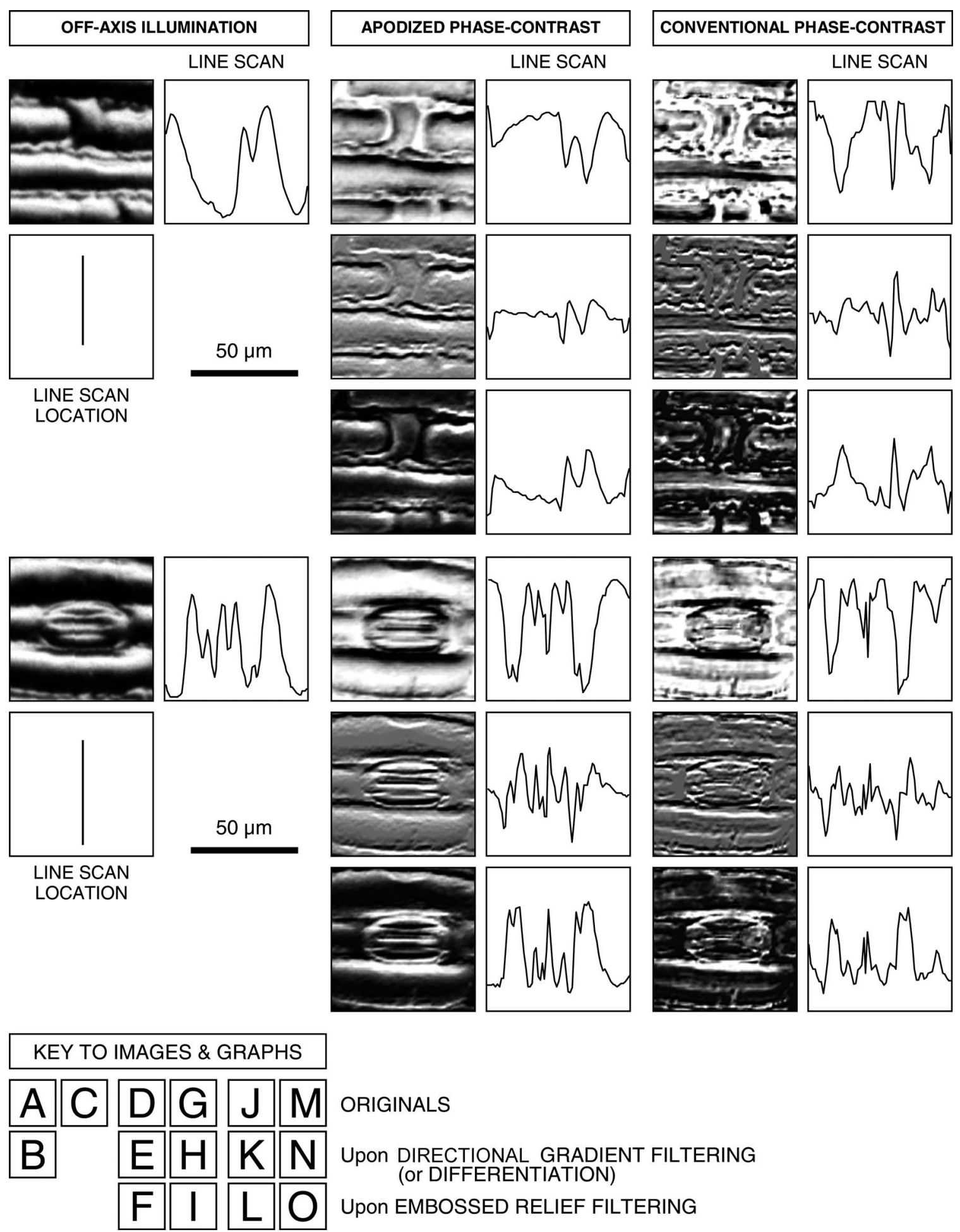

Fig. 4 Correlation between off-axis illumination and apodized phase-contrast images, as demonstrated with cutout details marked in Fig. 3. Note the similarity between the off-axis illumination (a) and appropriately filtered apodized phase-contrast (f) images. This contrasts with the very different visual impression exerted by the original, unfiltered images (d). The conventional phase-contrast images (j) suffer from extensive halo/ shade-off artifacts and make image filtering meaningless. Images: Original images (a), (d), and (j) were processed by directional gradient filtering [vertical differentiation, $(\mathrm{e})$ and $(\mathrm{k})$ ] or directional gradient filtering combined with luminance inversion [embossed relief filtering, $(\mathrm{f})$ and $(\mathrm{l})$; $(f)$ $=(e)$ minus $(d) ;(I)=(k)$ minus $(j)]$. Graphs: Each graph represents a luminance profile (0 to 255$)$ along a line positioned in the corresponding image as shown in box (b) (top to bottom scan). Grayscale in all graphs, 0 to 300. 


\section{OFF-AXIS ILLUMINATION}

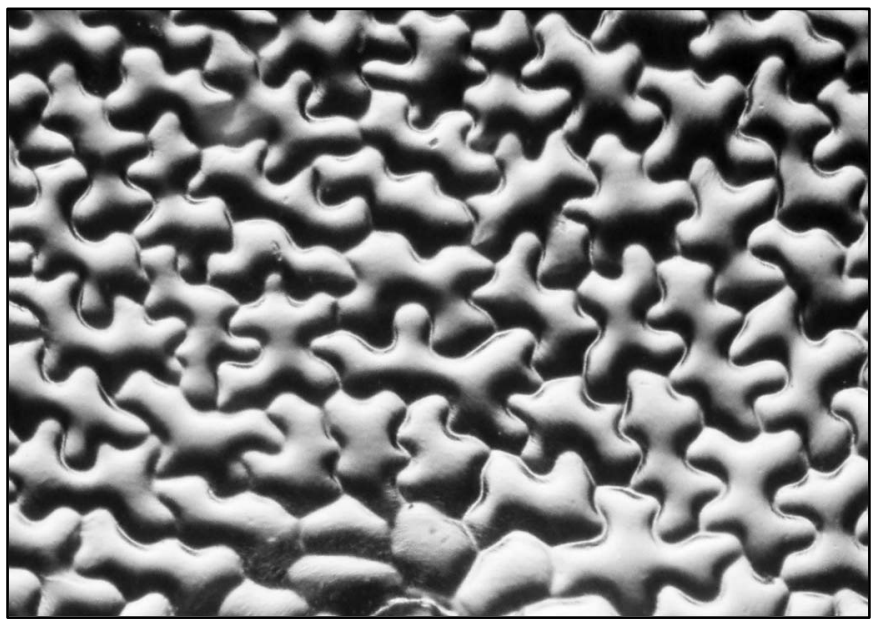

(a)

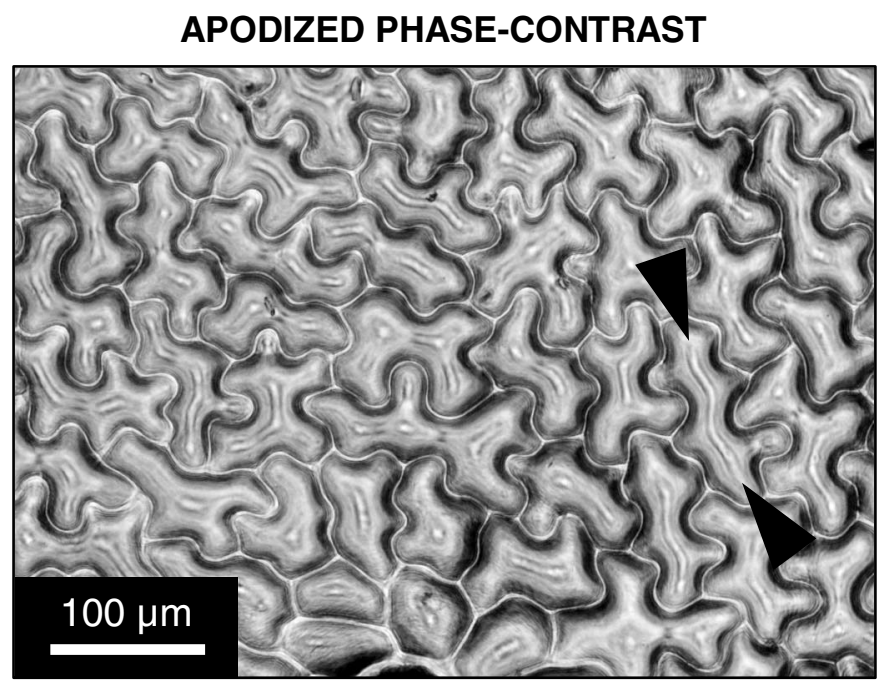

(c)

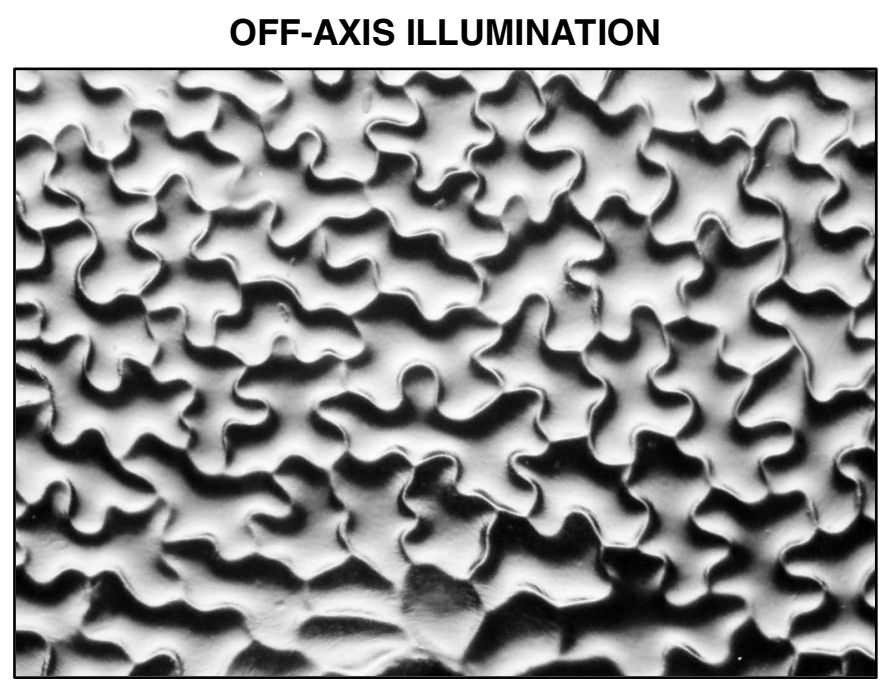

(b)

\section{CONVENTIONAL PHASE-CONTRAST}

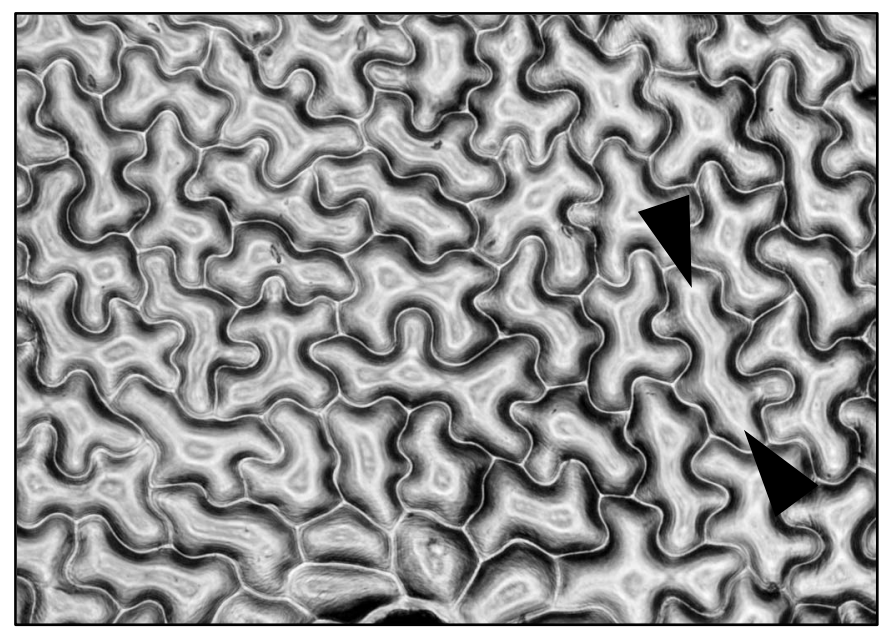

(d)

Fig. 5 Phase specimen of medium optical thickness and uniform refractive index (leaf replica from catchweed, Galium aparine). Panels (a) and (b); Note the strong quasi-3D effect and overall impression that is dependent on the choice of the apparent direction of the off-axis illumination (left, from above; right, from below). To most observers, the cell shapes appear either convex (left) or concave (right); invert the page to experience impression reversal. Panels (c) and (d): The halo/shade-off artifacts (arrowheads) present in the conventional phase-contrast image are noticeably reduced in the apodized phase-contrast one. See the text for an explanation. Phase-contrast type: positive.

Upon electronic color separation, the strongest color signal (green) was converted to grayscale (0 to 255) and contrast optimized; the grayscale values of $0 \%$ to $3 \%$ of the brightest and $0 \%$ to $3 \%$ of the darkest pixels were adjusted to 255 and 0 , respectively, and the histogram of the remaining $94 \%$ to $100 \%$ of pixels was expanded to cover the entire 0 to 255 grayscale range (linear expansion). This operation guarantees optimal print reproduction. However, it also slightly distorts the shape of image histograms. For this reason, the luminance profiles [Fig. 4, description follows], were obtained in images where the contrast was optimized by strictly using $100 \%$ (rather than, for example, 94\%) of image pixels, as described previously.

The contrast-optimized microscopic images shown in Fig. 3 were converted to quasi-3D rendering diagrams and video sequences (Video 1) showing the diagrams at a range of angles. The "3D-surface" function of Analysis Pro software was employed. The contrast in the still video images shown in Video 1 was optimized again.

The luminance profiles (line scans) were determined, at $98 \times 98$ pixel resolution, in selected cutout details from Fig. 3. These are presented in Figs. 4(a), 4(d), and 4(j), the precise location of the line scans in Fig. 4(b), and the profiles themselves in Figs. 4(c), 4(g), and 4(m). Top to bottom direction in the images corresponds to left to right direction in the graphs. The orientation of each line scan was always perpendicular to the edge of the central part of the relief diaphragm, that is, parallel to the apparent direction of illumination (north to south direction in Figs. 3 and 4).

Such profiles were also determined, in the same locations, in the apodized and conventional phase-contrast images 


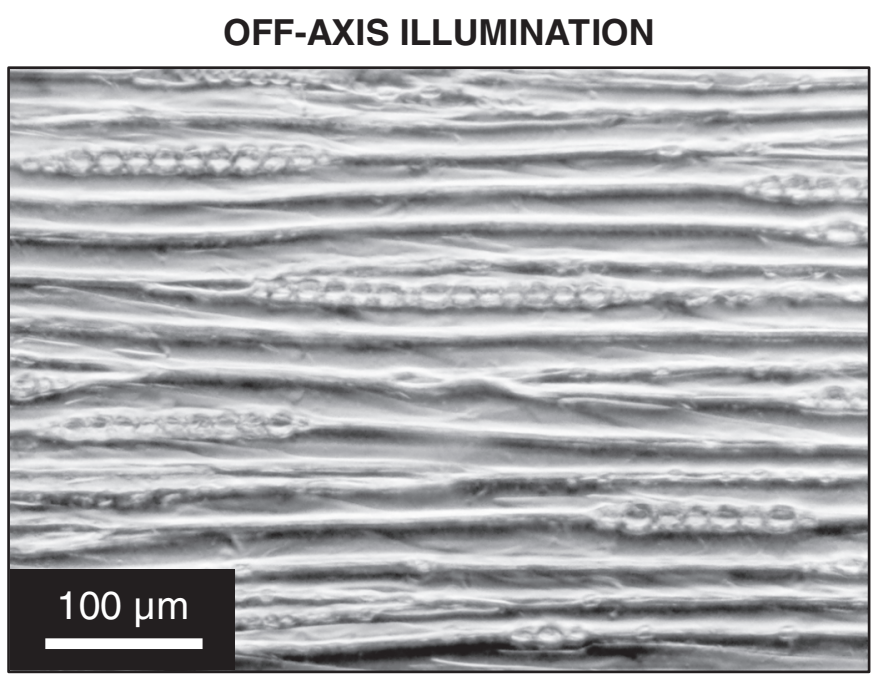

(a)

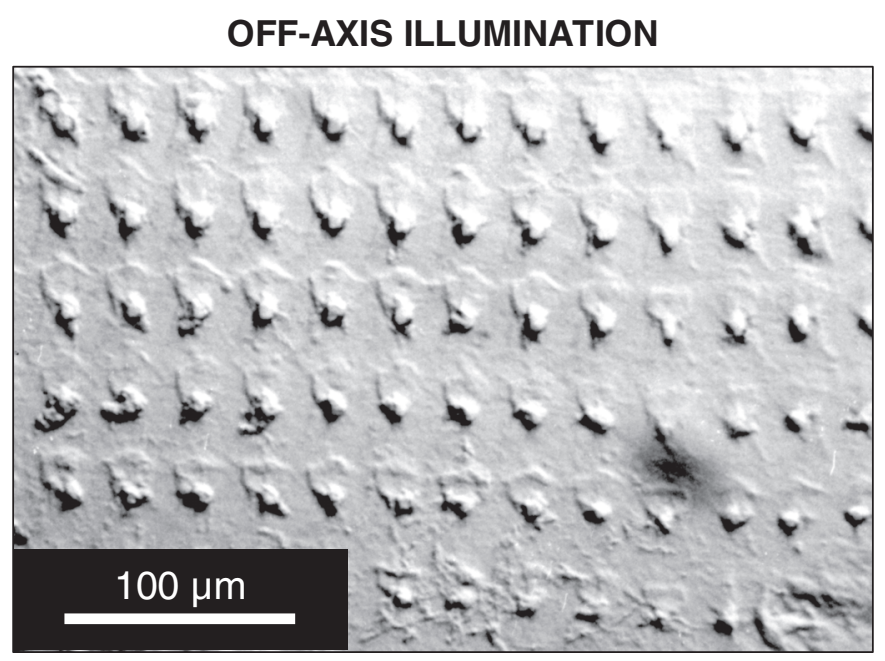

(c)

\section{APODIZED PHASE-CONTRAST}

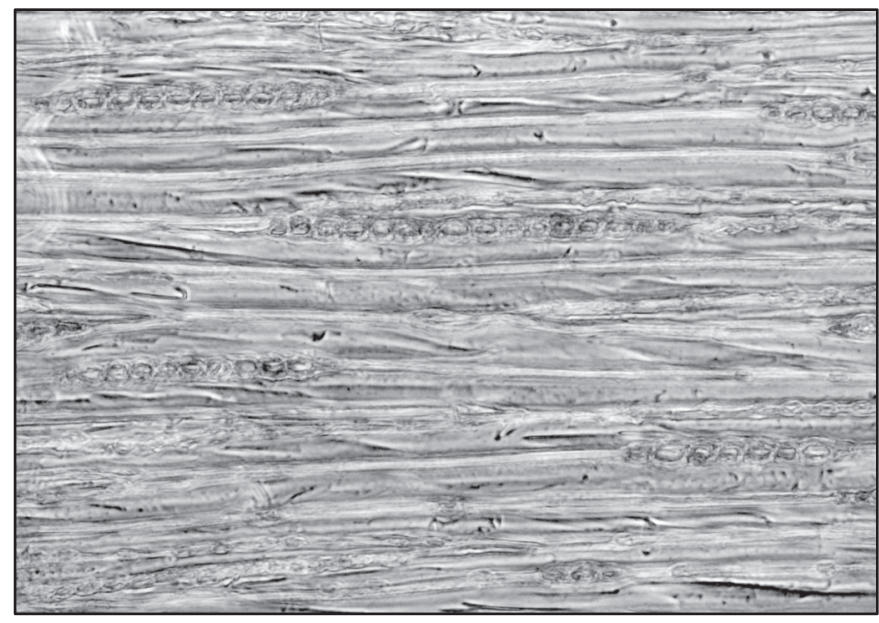

(b)

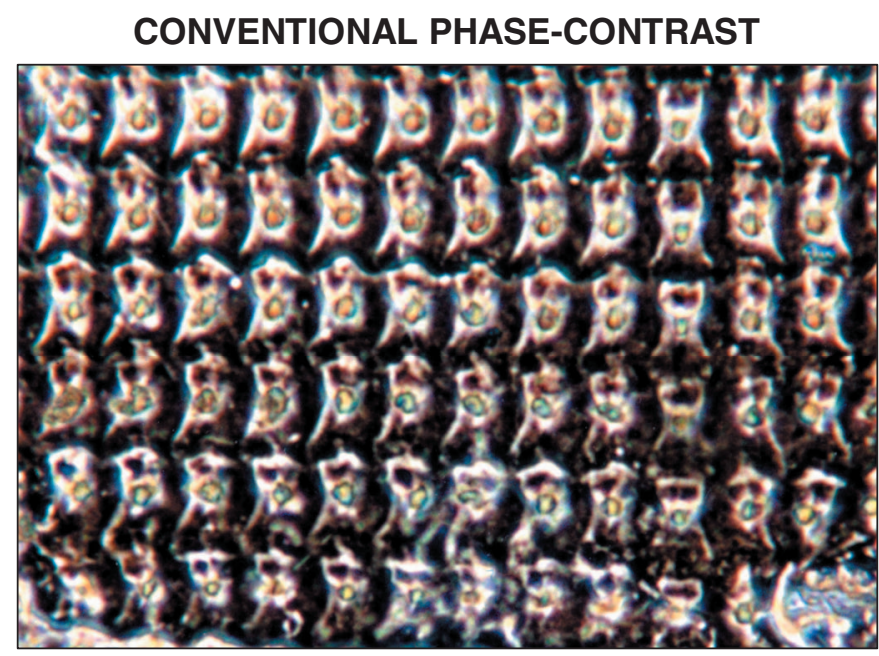

(d)

Fig. 6 Phase specimens with extreme values of optical thickness. Panels (a) and (b) (thick specimen): Longitudinal section through the wood of Norway spruce (Picea abies). Note that the off-axis illumination yields better contrast than phase-contrast. Panels (c) and (d) (thin specimen): The thinnest, still growing portion of Spanish slug's radula, as an example of a specimen in which the phase-contrast yields much higher contrast than the off-axis illumination. Phase-contrast type: positive (b) and negative (d).

[Figs. 4(d) and 4(j), respectively] previously processed, at $98 \times 98$ pixel resolution, by two kinds of filtering, as described in Secs. 2.4.1 and 2.4.2.

\subsubsection{Directional gradient filtering (or differentiation)}

The original image [Figs. 4(d) and 4(j)] was trimmed on top by two pixels and the resulting image $(100 \times 98)$ shifted up by two pixels. From that image, the original one [Figs. 4(d) and $4(\mathrm{j})$ ] was subtracted and the resulting image $(100 \times 98)$ was trimmed on the right by two pixels to $98 \times 98$ [Figs. 4(e) and $4(\mathrm{k})$ ]. The corresponding luminance profile [Figs. 4(h) and $4(n)]$ represents a directional gradient between $(n+2)$ th and $n$th pixel rows, i.e., a vertical gradient at $(n+1)$ th pixel row. The step was chosen to be two pixels $(\sim 1.37 \mu \mathrm{m})$, about the same as the resolving power of the $\times 10 / 0.25 \mathrm{ob}$ jective used. This is a compromise between a greater spatial precision and a more efficient suppression of high spatial frequency noise present in the luminance profiles.

\subsubsection{Embossed relief filtering}

From the $98 \times 98$ image obtained in the previous step (differentiation) [Figs. 4(e) and $4(\mathrm{k})$ ], the original image [Figs. 4(d) and 4(j)] was subtracted again, after being (i) trimmed to $98 \times 98$ by $1 / 1 / 2$ pixels from top/bottom/right and (ii) shifted up by 1 pixel. The resulting images and corresponding luminance profiles are shown in Figs. 4(f) and 4(l) and Figs. 4(i) and 4(o), respectively.

The subtraction operation with any pair of images meant multiplication of each of the two images by $1 / 2$, subtraction, and application of a 127 offset to prevent the grayscale intensity becoming negative or saturating at 255 level. In order for 
all seven images of the same detail [Figs. 4(a), 4(d)-4(f), and 4(j)-4(1)] to match, Fig. 4(a) was trimmed to $98 \times 98$ in the same way as Fig. 4(d) and 4(j) (by $1 / 1 / 2$ pixels from top/bottom/right).

All electronic operations with the images were performed with Analysis Pro version 3.1 or 3.2 (Soft Imaging Solutions $\mathrm{GmbH}$, Münster, Germany), Adobe Photoshop version 7.0.1 CE (San Jose, California), or with ImageJ version 1.40g (National Institutes of Health, Bethesda, Maryland).

\section{Results}

The coachgrass leaf replica images and corresponding quasi-3D (pseudo-relief) surface rendering diagrams are shown in Fig. 3 and Video 1, respectively. The diagrams can also be inspected at a range of viewing angles in online videos (1 deg increment animation). Selected image details, marked in Fig. 3 by boxes, are shown at an expanded scale in Figs. 4(a), 4(d), and 4(j).

Although the information contents in the off-axis illumination and apodized phase-contrast images is comparable, the conventional phase-contrast ones suffer from extensive halo/ shade-off artifacts that overwhelmingly blur the structural details, especially edges. This demonstrates the efficiency of artifact reduction by apodization.

These points are further documented in images processed by directional gradient [Figs. 4(e) and 4(k)] and embossed relief [Figs. 4(f) and 4(l)] filtering. The latter combines the directional gradient-filtered (differentiated) images with those in which the intensities were inverted (positive-negative). Note the similarity between Figs. 4(a) (original images) and 4(f) (embossed relief images).

The filtered apodized phase-contrast images [Figs. 4(e) and 4(f)] feature a relief impression to some extent, as does the off-axis illumination image [Fig. 4(a)]. In many cases, a mere inversion of the luminance (positive-negative) was nearly sufficient to convert the original apodized phase-contrast images [Fig. 4(d)] to those similar to the off-axis illumination ones [Fig. 4(a)].

Indeed, both off-axis illumination and apodized phasecontrast imaging modes inherently suppress low spatial frequencies, thus highlighting edges/contours. Apodization also "deblurs" the images by eliminating the halo/shade-off artifacts associated with large optical path difference steps/ gradients (edges). On the other hand, no meaningful information can be extracted from the filtered conventional phasecontrast images as the structure is either "flattened" [Fig. 4(k)] or "scrambled" [Fig. 4(1)].

The luminance profiles, performed vertically as line scans, located as shown in Fig. 4(b), are also presented [Figs. 4(c), $4(\mathrm{~g})$, and $4(\mathrm{~m})]$. The profiles of the filtered images are shown in Figs. 4(h) and 4(i) (apodized phase-contrast) and Figs. 4(n) and $4(\mathrm{o})$ (conventional phase-contrast). The luminance profiles obtained in the off-axis illumination images [Fig. 4(c)] are comparable to some extent to those obtained in the filtered apodized phase-contrast ones [Fig. 4(i)]. As mentioned in Sec. 1, the graphs shown in Figs. 4(c) and 4(h) may theoretically match. In reality, however, the fit between them is far from perfect, and at least part of the reason are some of the following factors:

1. Specimen thickness; the dependency between the lumi- nance value in the phase-contrast image and the OPD is nearly linear only in certain OPD ranges (a fraction of the wavelength). ${ }^{12,13}$

2. The luminance values in the off-axis illumination images are in fact not exactly proportional to the OPD differentials. A special filter at the objective back focal (Fourier transform) plane would have to be used to achieve a closer correspondence. ${ }^{11}$

3. Before the luminance profiles were obtained in the two cutout details shown in Fig. 4, the images were electronically processed (contrast adjusted), as described in Sec. 2. The luminance profiles along one line were thus affected to some extent by the rest of the cutout details; the correspondence was somewhat better before the contrast was "adjusted" (data not shown).

Another set of images from a specimen of medium thickness (leaf replica from catchweed) is shown in Fig. 5. Two opposite apparent directions of illumination were chosen in the off-axis illumination mode; note the strong illusion of convex/concave shapes conveyed by shading that serves as a visual cue to depth structure. The quasi-3D (pseudo-relief) impression is particularly strong in this "jigsaw-puzzle" specimen, perhaps owing to the high density of edges (cell borders) where the shading patterns are most prominent.

The conventional phase-contrast image in Fig. 5 is partly blurred by the shade-off artifacts spreading along cell axes. These artifacts are mostly eliminated by the apodized phasecontrast; only thin 'ridges' remain (arrowheads). The image areas that are bright in the conventional phase-contrast but turn dark in the apodized phase-contrast roughly correspond to the OPD of $\lambda / 6+n \lambda$; compare vectors $\vec{e}$ (bright) and $\vec{g}$ (dark) in Fig. 2. The ridges may thus be regarded as optical thickness contour lines below which the image is free from the halo/shade-off artifacts.

The complementarity of the off-axis illumination and apodized or conventional phase-contrast modes is demonstrated in rather thick and thin specimens (Fig. 6). The section through the wood of Norway spruce (thick specimen) is imaged much better in the off-axis illumination mode [Fig. $6(\mathrm{a})$. The effect of apodization is rather moderate in this specimen (image not shown), as theoretically predictable (Fig. 2 and elsewhere in the paper). In the Spanish slug's radula (thin specimen), the off-axis illumination mode is apparently rather inefficient [Fig. 6(c)]; the contrast and quasi-3D effect are very weak. At the same time, the halo/shade-off artifacts are almost absent even in the conventional phase-contrast image [Fig. 6(d)]. In a thicker part of the same specimen, the opposite is the case, as shown elsewhere. ${ }^{31}$

\section{Discussion}

Apodized phase-contrast makes it possible to reliably examine under the phase-contrast microscope thicker objects than in the conventional phase-contrast. These include large cells, ${ }^{28,33}$ embryos, ${ }^{28}$ and even small organisms, for example, living roundworms ${ }^{22}$ (Caenorhabditis elegans, a popular model in developmental biology studies, $\sim 50 \mu \mathrm{m}$ in diameter). Tomographic $3 \mathrm{D}$ reconstructions ${ }^{27}$ have also been performed. ${ }^{33}$ Development has been underway to further reduce the halo/shade-off artifacts in the images, for example, with graded-transmittance apodization annuli ${ }^{21}$ or a pupil- 
projection (optical relay) setup. ${ }^{26}$ Much like in, for example, Nikon's interference-phase attachment described elsewhere, ${ }^{12,13}$ the latter configuration makes the phase plate physically accessible even in high-magnification objectives, allowing us to fine-tune its parameters to the object under investigation. $^{23-25}$

Related designs presented so far include (i) special phase plates, such as those originally designed for tracing irregularities on lens surfaces, ${ }^{34}$ including antireflecting soot layers ("anoptral contrast" from Reichert Co.); $;^{12,13,35,36}$ (ii) a combination of anoptral contrast with dark field illumination; ${ }^{37}$ (iii) "variable phase-contrast" systems equipped with, for example, two separate phase annuli ("Phv" objectives from Zeiss $)^{38,39}$ or a condenser enabling a continuous change of the diameter of the condenser annulus ${ }^{40}$ (in a way, a modification of so-called universal phase condenser). A more detailed account of these designs can be found elsewhere, ${ }^{12,13}$ including a combination of phase-contrast with interference microscopy.

The off-axis illumination mode is capable of introducing into the images the quasi-3D effect, thus facilitating their comprehension by the human visual system. ${ }^{41,42}$ Corresponding physiological activity has been demonstrated directly in human and monkey brains, either by functional magnetic resonance imaging ${ }^{43,44}$ or by recording single neuronal activity. ${ }^{45}$

The quasi-3D effect is perceived owing to so-called visual cues to depth structure (depth cues). They make it easier for the image, as detected on the retina in the eye, to be correctly interpreted by the visual cortex in human brain. Apart from motion cues and the binocular parallax, where both eyes are involved, there are also monocular cues that include shading, texture, occlusion, and contours (silhouette). Not all of these cues are present in every quasi-3D image, and not always are the different types of cues nonconflicting ("consistent-cue stimulus"). ${ }^{42,44}$

Shading as a visual cue should not be confused with the shade-off or shading-off artifact referred to throughout the present paper in relation to the phase-contrast microscopy. The shading cue is inherently present in the off-axis illumination or Hoffman modulation contrast ${ }^{5,6}$ images, hence the term "schlieren imaging" (see Sec. 1 for details), implying the presence of "streaks" (shading patterns) in the images. The same applies to the differential interference contrast after Nomarski ${ }^{4}$ using an altogether different optical principle. All of these imaging modes deliver quasi-3D (pseudo-relief) images and so do certain postdetection (image shift and subtraction) procedures ${ }^{16-18}$ and online approaches using split detectors, ${ }^{46}$ eliminating the need for any postdetection processing altogether. The latter thus essentially imitate the visual processing in the human brain.

In the phase-contrast images, on the other hand, shading as a visual cue to depth structure is absent, although a version of the phase-contrast microscope that would bring about the quasi-3D effect by using noncircular phase strips was described a long time $\mathrm{ago}^{2}$ and various other modifications have been proposed since. ${ }^{12,13,47-52}$ A commercially available one called VAREL (Variable Relief Contrast) ${ }^{53}$ has been manufactured by Zeiss; its origin lies in a system allowing a switch between the phase-contrast and relief (off-axis) illumination, without having to change the objective lens. ${ }^{49}$ Yet another type has been proposed with the aim to improve the efficiency of the optical microlithography process. ${ }^{54}$

The quasi-3D (pseudo-relief) effects were also achieved in conjunction with laser scanning (confocal) microscopy by using half-aperture stops ${ }^{55,56}$ or split detectors, ${ }^{46,57,58}$ or by shifting and subtracting images obtained by slight under- and overfocusing. ${ }^{59}$ Despite the absence of any phase-shifting elements the quasi-3D approach is often referred to as "differential phase-contrast" "55,57,58 or (incorrectly) "differential interference contrast," $" 59$ which only highlights the complementary nature of the off-axis illumination and phasecontrast imaging.

Despite the advantages of directly obtaining the quasi-3D (pseudo-relief) images, it should be borne in mind that certain structures may easily be misinterpreted when using the offaxis illumination alone [see, for example, the top cutout detail in Figs. 4(a) and 4(d)]. Owing to a greater depth of field and the presence in the images of the visual cues to depth structure, this mode may be useful in the initial screening of a specimen, before selected details are subjected to a rigorous examination under the apodized phase-contrast.

\section{Conclusion}

The present paper describes the off-axis illumination and the apodized phase-contrast microscopy as two complementary phase-imaging modes. Their combination is particularly suitable to examine specimens of medium optical thickness (e.g., large cells or cell clusters) in which the two modes overlap in terms of phase visualization capability. From the viewpoint of image information contents alone, the two image types are comparable ("off-line machine vision"). Although the structure itself may often be more faithfully represented in the apodized phase-contrast images, an important visual cue to depth structure (shading) is present in the off-axis illumination images only, thus enabling an instant image interpretation by an experimentalist ("online human vision"). This may be of importance, for example, when observing highly motile cells or in routine screening of large specimen sets. The points outlined in the present paper could be demonstrated by using halo/shade-off artifact reduction in phase-contrast images by apodization.

\section{Acknowledgments}

The authors are grateful to Dr. Kaoru Katoh (National Institute of Advanced Industrial Science and Technology-AIST, Tsukuba, Japan) for helpful discussions. Mr. Hiroyasu Tanaka (Nikon Instruments Company's BioScience Division, Kawasaki City, Japan) and the staff, and Mr. Daniel Mack (Nikon s.r.o., Prague) kindly enabled acquisition of the positive phase-contrast images on their equipment. Many thanks are due to Drs. Jiří Janáček, Petr Bouř, Lucie Kubínová, and Oldřich Benada (all at Czech Academy of Sciences, Prague) for their advice about electronic image formats, and to the staff at Fotomedia a.s. in Prague for their extra effort. Institute of Physiology was supported by a framework grant No. AV0Z50110509 and a program grant No. LC06063. A partial support to RP is gratefully acknowledged (public funds, Czech Academy of Sciences' framework grant, Ref. No. AV0Z50200510; private funds, FEI/Hitachi/JEOL-sponsored Scholarship for Young Scientists to visit Japan). 
Pelc, Hostounský, and Otaki: Correlation between off-axis illumination...

\section{References}

1. F. Zernike, "Beugungstheorie des Schneidenverfahrens und seiner verbesserten Form, der Phasenkontrastmethode," Physica (Amsterdam) 1(8), 689-704 (1934).

2. F. Zernike, "Phase contrast, a new method for the microscopic observation of transparent objects," Physica (Amsterdam) 9(7), 686-698 (1942); 9(10), 974-986 (1942).

3. F. Zernike, "How I discovered phase contrast," Science 121(3141), 345-349 (1955).

4. G. Nomarski, "Microinterféromètre différentiel à ondes polarisées," J. Phys. Radium 16(7), S9-S13 (1955).

5. G. S. Settles, Schlieren and Shadowgraph Techniques, SpringerVerlag, Berlin (2001).

6. R. Hoffman, "The modulation contrast microscope: principles and performance," J. Microsc. (Oxford) 110(Pt3), 205-222 (1977).

7. A. Töpler, "Ueber die Methode der Schlierenbeobachtung als mikroskopisches Hülfsmittel, nebst Bemerkungen zur Theorie der schiefen Beleuchtung," (Poggendorff's) Annalen der Physik und Chemie (Leipzig) 127(4), 556-580 (1866), available online in Ann. Phys. 203(4), 556-580 (1866).

8. E. Abbe, "Ueber einen neuen Beleuchtungsapparat am Mikroskop," (Schultze's) Archiv für Mikroskopische Anatomie (Bonn) 9, 469-480 (1873).

9. Z. Hostounský and R. Pelc, "An efficient way of high-contrast, quasi-3D cellular imaging: Off-axis illumination," J. Biochem. Biophys. Methods 68(1), 23-30 (2006).

10. R. A. Sprague and B. J. Thompson, "Quantitative visualization of large variation phase objects," Appl. Opt. 11(7), 1469-1479 (1971).

11. E. Tajahuerce, T. Szoplik, J. Lancis, V. Climent, and M. Fernandez, "Phase-object fractional differentiation using Fourier plane filters," Pure Appl. Opt. 6(4), 481-490 (1997).

12. M. Pluta, "Non-standard methods of phase contrast microscopy," in Advances in Optical and Electron Microscopy, vol. 6, R. Barer, V. E. Coslett, Eds., pp. 49-133, Academic Press, London (1975).

13. M. Pluta, "Phase contrast microscopy," in Advanced Light Microscopy, vol. 2; Specialized Methods, Chapter 5, pp. 1-90 and 385-388, Elsevier \& PWN-Polish Scientific Publishers, Warszawa (1989).

14. T. Otaki, "Halo reduction technique in phase contrast microscope," Opt. Rev. 8(4), 284-286 (2001).

15. J. E. Rhodes, Jr., "Microscope imagery as carrier communication," $J$. Opt. Soc. Am. 43(10), 848-852 (1953).

16. S. Inoué, Video Microscopy, Plenum Press, New York (1986).

17. E. D. Barone-Nugent, A. Barty, and K. A. Nugent, "Quantitative phase-amplitude microscopy I: optical microscopy," J. Microsc. (Oxford) 206(Pt.3), 194-203 (2002).

18. P. J. McMahon, E. D. Barone-Nugent, B. E. Allman, and K. A. Nugent, "Quantitative phase-amplitude microscopy II: differential interference contrast imaging for biological TEM," J. Microsc. (Oxford) 206(Pt3), 204-208 (2002).

19. J. Rheinberg, "On the influence on images of gratings of phase difference amongst their spectra," J. R. Microsc. Soc. Pt. 4, 388-390 (1904), and Pt. 2, 152-155 (1905).

20. T. Otaki, "Artifact halo reduction in phase contrast microscopy using apodization," Opt. Rev. 7(2), 119-122 (2000).

21. T. Otaki, "Phase contrast observation device," U.S. Patent No. US6,317,261 B1 (2001).

22. T. Otaki, K. Katoh, and F. Yoshida, "Phase objects observing methods for video microscopy: apodized phase contrast, differential interference contrast with short shear and polarizing-microscope using liquid crystals" (in Japanese), Electron Microsc. 37(Suppl. 2), 105-108 (2002).

23. T. Otaki, M. Suzuki, and K. Katoh, "Apodized phase contrast microscopy yields refined dynamic images of organelles in living cultured cells," (in Japanese), in Proc. 29th Kogaku Symposium, p. 43-46 (2004).

24. K. Katoh, T. Otaki, and M. Suzuki, "Dynamics of actin cytoskeleton revealed in living cells with a novel phase contrast system," Presented at 44th Annual Meeting, Am. Soc. Cell Biol., 4-8 December 2004, Washington, D.C., Abstract L103.

25. T. Otaki and K. Katoh, "Apodized phase-contrast microscopy, theory and images," in Proc. 16th Int. Microscopy Congress (IMC16), p. 98 (2006) CD-ROM.

26. K. Katoh and T. Otaki, "Imaging living samples with apodized phase contrast microscopy, a novel phase contrast microscopy," in Proc. 16th Int. Microscopy Congress (IMC16), p. 64 (2006). CD-ROM.
27. T. Otaki, "Phase contrast microscope." Japanese Patent Application No. JP2,006,163,090A (2006).

28. T. Otaki, C. D. Howard, and M. W. Davidson, "Halo reduction with apodized phase contrast," Nikon's on-line "Microscopy University" (includes interactive Java tutorials), available at: http:// www.microscopyu.com/articles/phasecontrast/apodized.html.

29. C. L. Curl, C. J. Bellair, T. Harris, B. E. Allman, P. J. Harris, A. G. Stewart, A. Roberts, K. A. Nugent, and L. M. D. Delbridge, "Refractive index measurement in viable cells using quantitative phaseamplitude microscopy and confocal microscopy," Cytometry, Part A 65A(1), 88-92 (2005).

30. C. L. Curl, T. Harris, P. J. Harris, B. E. Allman, C. J. Bellair, A. G. Stewart, and L. M. D. Delbridge, "Quantitative phase microscopy: a new tool for measurement of cell culture growth and confluency in situ," Pfluegers Arch. 448(4), 462-468 (2004).

31. Z. Hostounský and R. Pelc, "Phase-contrast versus off-axis illumination: is a more complex microscope always more powerful?," $A d v$. $\begin{array}{lllll}\text { Physiol. Educ. 31(2), 232-235, } 281 \quad \text { (2007); http:// } & \end{array}$ advan.physiology.org/cgi/reprint/31/2/232.

32. Z. Hostounský, "Osvětlovací soustava pro mikroskopické pozorování průhledných neabsorbujících objektü," Czech Utility Model No. CZ4925U (1996).

33. H. Kobayashi, I. Ishimaru, T. Yasokawa, K. Ishizaki, S. Kuriyama, T. Masaki, S. Nakai, K. Takegawa, and N. Tanaka, "Three-dimensional phase-contrast imaging of single floating cells," Appl. Phys. Lett. 89(24), 241117 (2006).

34. B. Lyot, "Procédés permettant d'étudier les irrégularités d'une surface optique bien polie," C. R. Hebd. Seances Acad. Sci. 222(14), 765-768 (1946).

35. A. Wilska, "A new method of light microscopy," Nature (London) 171(4347), 353 (1953).

36. A. Wilska, "Observations with the anoptral microscope," Mikroskopie 9(1/2), 1-80 (1954).

37. V. Soran and B. Diaconeasa, "The ultra-anoptral microscope-an improvement of Wilska's method," Naturwiss. 44(17), 465-465 (1957).

38. H. Beyer, "Untersuchungen über den Einfluß der Gestalt der Aperturblende auf die mikroskopische Abbildung beim Phasenkontrastverfahren," in Jenaer Jahrbuch 1953, pp. 162-209, VEB Carl Zeiss, Jena \& VEB Gustav Fisher, Jena, Germany (1953).

39. H. Beyer, Theorie und Praxis des Phasenkontrastverfahrens, P. Görlich, Ed., vol. 17, (Technisch-Physikalische Monographien), Akademische Verlagsgesellschaft Geest Portig K.-G., Leipzig (1965).

40. M. L. Peshkov, "A new type of objectives for anoptral microscopy, utilising an opening, and a brief analysis of the principle of their operation" (in Russian), Usp. Sovrem. Biol. 40(3), 372-378 (1955).

41. J. Sun and P. Perona, "Where is the sun?," Nat. Neurosci. 1(3), 183184 (1998).

42. A. E. Welchman, A. Deubelius, V. Conrad, H. H. Bülthoff, and Z. Kourtzi, "3D shape perception from combined depth cues in human visual cortex," Nat. Neurosci. 8(6), 820-827 (2005).

43. G. K. Humphrey, M. A. Goodale, C. V. Bowen, J. S. Gati, T. Vilis, B. K. Rutt, and R. S. Menon, "Differences in perceived shape from shading correlate with activity in early visual areas," Curr. Biol. 7(2), 144-147 (1997).

44. M. E. Sereno, T. Trinath, M. Augath, and N. K. Logothetis, "Threedimensional shape representation in monkey cortex," Neuron 33(4), 635-652 (2002).

45. K.-I. Tsutsui, H. Sakata, T. Naganuma, and M. Taira, "Neural correlates for perception of 3D surface orientation from texture gradient," Science 298(5592), 409-412 (2002).

46. D. K. Hamilton, C. J. P. Sheppard, and T. Wilson, "Improved imaging of phase gradients in scanning optical microscopy," J. Microsc. (OXford) 135(Pt3), 275-286 (1984).

47. F. Bretschneider and P. F. M. Teunis, "Reduced-carrier singlesideband microscopy: a powerful method for the observation of transparent microscopic objects," J. Microsc. (Oxford) 175(Pt2), 121-134 (1994).

48. M. Pluta, "Stereoscopic phase contrast microscope," Microscope 16 (1st quarter issue), 32-36 (1968).

49. H. Piller and K. Weber, "Device for alternately realizing the microscopic observation in phase contrast or in relief," European Patent No. EP0,069,263 (1983).

50. H. Takaoka, "Optical microscope which has optical modulation elements," U.S. Patent Nos. US5,969,853 (1999) and US6,130,776 (2000). 
51. J. Brief, "Minimal dezentriert Phasenkontrast-eine sehr einfache methode zur Kontrast-verbesserung bei hoher Vergrößerung," Mikrokosmos 94(4), 203-205 (2005).

52. J. Piper, "Relief phasenkontrast—eine universell einsetzbare modifikation des phasenkontrast mit verbesserter Bildqualität," Mikrokosmos 96(4), 223-229 (2007).

53. B. Hohman and E. Keller, "VAREL: a new contrasting method for microscopy," Biomed. Prod., 3 (January 2001), http:// www.zeiss.com/micro.

54. M. Erdelyi, Z. Bor, J. R. Cavallaro, G. Szabo, W. L. Wilson, C. Sengupta, M. C. Smayling, and F. K. Tittel, "Enhanced microlithography using combined phase shifting and off-axis illumination," Jpn. J. Appl. Phys., Part 2 34(12A), L1629-L1631 (1995).

55. P. Török, C. J. R. Sheppard, and Z. Laczik, "Dark-field and differential phase contrast imaging modes in confocal microscopy using a half-aperture stop," Optik (Jena) 103(3), 101-106 (1996).
56. K. K. Chu, R. Yi, and J. Mertz, "Graded-field autoconfocal microscopy," Opt. Express 15(5), 2476-2489 (2007).

57. M. R. Atkinson, A. E. Dixon, and S. Damaskinos, "Surface-profile reconstruction using reflection differential phase-contrast microscopy," Appl. Opt. 31(31), 6765-6771 (1992).

58. W. B. Amos, S. Reichelt, D. M. Cattermole, and J. Laufert, "Reevaluation of differential phase contrast (DPC) in a scanning laser microscope using a split detector as an alternative to differential interference contrast (DIC) optics," J. Microsc. (Oxford) 210(Pt2), $166-175$ (2003).

59. S. H. Cody, S. D. Xiang, M. J. Layton, E. Handman, M. H. C. Lams, J. E. Layton, E. C. Nice, and J. K. Heath, "A simple method allowing DIC imaging in conjunction with confocal microscopy," J. Microsc. (Oxford) 217(Pt3), 265-274 (2005). 